\title{
ON A PROBLEM OF C. E. SHANNON IN GRAPH THEORY
}

\section{ROSENFELD ${ }^{1}$}

1. Introduction. We consider in this paper only finite nondirected graphs without multiple edges and we assume that on each vertex of the graph there is a loop, i.e. each vertex of the graph is connected to itself by an edge. O. Ore in [2] raised the following problem: Given a finite graph $F$, what are the necessary and sufficient conditions on $G$ in order that

$$
\mu(G \times H)=\mu(G) \cdot \mu(H) \quad \text { for every finite graph } H .
$$

A partial answer is given by the following theorem due to Shannon [3]:

Theorem 1 (Shannon). If there exists a preserving function $\sigma$ defined on $G$ such that $\sigma(G)$ is an independent set of vertices in $G$ then (1) holds for every finite graph $H$.

For a proof of Shannon's theorem see for example [1], [3].

Shannon proved the sufficiency of his condition only. Our main result is a necessary and sufficient condition under which (1) always holds (Theorem 2) and to show that Shannon's condition is not necessary $(\$ 4)$. Our condition will be given in terms of linear programming.

2. Definitions and notations. By an independent set of vertices in a graph $G$ we mean a subset of vertices such that no two different vertices in the subset are joined by an edge in $G$. The maximal number of independent vertices in a graph $G$ will be denoted by $\mu(G)$. A clique in a graph $G$ is a complete subgraph of $G$ (i.e. a set of vertices each pair of which are connected by an edge) which is not contained in any other complete subgraph of $G$. $\operatorname{Ver}(G)$ will denote the set of vertices of $G$. A function $\sigma: G \rightarrow G$ will be called preserving if $g \rightarrow g^{\prime}$ $\Rightarrow \sigma(g) \rightarrow \sigma\left(g^{\prime}\right)$ (where $g \rightarrow g^{\prime}$ means that $g$ is not joined by an edge to the vertex $g^{\prime}$ ). The cartesian product of two graphs is a graph denoted by $G \times H$ defined as follows:

$\operatorname{Ver}(G \times H)=\operatorname{Ver}(G) \times \operatorname{Ver}(H),(g h) \rightarrow\left(g^{\prime} h^{\prime}\right)$ iff $g \rightarrow g^{\prime}$ and $h \rightarrow h^{\prime}$.

A graph $G$ for which the equality (1) always holds will be called universal.

Received by the editors February 3, 1966.

1 This paper is a part of the author's Ph.D. thesis to be submitted to the Hebrew University, Jerusalem. The author wishes to express his gratitude to M. O. Rabin for his helpful suggestions. 
3. Let $G$ be a finite graph. $\operatorname{Ver}(G)=\left\{g_{1} \cdots g_{n}\right\}$. Let $\left\{C_{1} \cdots C_{s}\right\}$ be a fixed ordering of all the different cliques of $G$. Define $\alpha_{i}^{(j)}$ as follows:

$$
\begin{aligned}
\alpha_{i}^{(j)} & =1, & & g_{i} \in C_{j}, \\
& =0, & & g_{i} \notin C_{j} .
\end{aligned}
$$

Let $P_{G}=\left\{\left(x_{1} \cdots x_{n}\right) \mid \sum_{i=1}^{n} \alpha_{i}^{(j)} x_{i} \leqq 1, x_{i} \geqq 0,1 \leqq j \leqq s\right\}$.

TheOREM 2. A finite graph $G$ is universal if and only if

$$
\max _{x \in P_{G}} \sum_{i=1}^{n} x_{i}=\mu(G), \quad x=\left(x_{1} \cdots x_{n}\right) .
$$

Proof. (i) Without loss of generality we may assume that $\left\{g_{1} \cdots g_{\mu(G)}\right\}=A$ is an independent set of vertices in $G$. Choose: $x_{i}=1,1 \leqq i \leqq \mu(G), x_{i}=0, i>\mu(G)$. Since no two vertices in $A$ are contained in the same clique it is obvious that for every $j$ we have:

$$
\sum_{i=1}^{n} \alpha_{i}^{(j)} x_{i} \leqq 1 \quad \text { while } \sum_{i=1}^{n} x_{i}=\mu(G) .
$$

Therefore we always have $\max \sum_{i=1}^{n} x_{i} \geqq \mu(G)$.

(ii) Suppose $G$ is not universal, i.e. there exists a graph $H$ such that

$$
\mu(G \times H)>\mu(G) \cdot \mu(H) .
$$

(It is obvious that $\mu(G \times H) \geqq \mu(G) \cdot \mu(H)$.) Let $A \subset G \times H$ be a maximal independent set of vertices in $G \times H$ (i.e. card $A=\mu(G \times H)$ ). Define $\quad A_{i}=\left\{h \mid\left(g_{i} h\right) \in A\right\}, \quad\left(A_{i} \subset H\right)$. Since $\quad\left(g_{i} h\right) \rightarrow\left(g_{i} h^{\prime}\right) \quad$ if $h \rightarrow h^{\prime}$ and $A$ is independent it follows that $A_{i}$ is an independent set of vertices in $H$ and therefore card $A_{i} \leqq \mu(H)$. Furthermore if $A_{i}^{\prime}$ $=\left\{\left(g_{i} h\right) \mid h \in A_{i}\right\}$ then card $A=\bigcup_{i=1}^{n} A_{i}^{\prime}$ and the union is disjoint. Now choose $x_{i}=(1 / \mu(H))$ card $A_{i}$, it is obvious that

$$
\sum_{i=1}^{n} x_{i}=\frac{1}{\mu(H)} \sum_{i=1}^{n} \operatorname{card} A_{i}^{\prime}=\frac{\mu(G \times H)}{\mu(H)}>\mu(G) .
$$

Let us show that, for every $j, \sum_{i=1}^{n} \alpha_{i}^{(j)} x_{i} \leqq 1$. If

$$
C_{j}=\left\{g_{i_{1}} \cdots g_{i_{k}}\right\} \Rightarrow \sum_{i=1}^{n} \alpha_{i}^{(j)} x_{i}=\sum_{l=1}^{k} x_{i_{l}} .
$$

Since $g_{i_{r}} \rightarrow g_{i_{t}}, 1 \leqq r, t \leqq k$, it follows that $\bigcup_{l=1}^{k} A_{i_{l}}$ is an independent set of vertices in $H$, and the union is disjoint, hence the following holds

$$
\mu(H) \sum_{l=1}^{k} x_{i}=\sum_{l=1}^{k} \operatorname{card} A_{i_{l}}=\operatorname{card}\left\{\bigcup_{l=1}^{k} A_{i_{l}}\right\} \leqq \mu(H) .
$$


Thus, (3) and (4) prove that our condition is necessary.

(iii) To prove the sufficiency of our condition, suppose that

$$
\max _{x \in P_{G}} \sum_{i=1}^{n} x_{i}>\mu(G) .
$$

Since $P_{G}$ is a polytope in the $n$-dimensional Euclidean space the maximum is obtained; furthermore, since the coefficients of the half spaces determining the polytope are nonnegative integers we may assume without loss of generality that all the components $\left(x_{1} \cdots x_{n}\right)$ of the maximizing point which can be chosen to be a vertex of $P_{G}$ are rational. Let $\beta$ be the least common multiplier of all the denominators of the $x_{i}$ 's (it is obvious that $\beta<n$ ! being the determinant of a matrix of order $n$ with 0 's and 1 's). Let $y_{i}=\beta \cdot x_{i}$, hence $\left\{y_{i}\right\}$ is a set of nonnegative in tegers satisfying

$$
\begin{gathered}
\sum_{i=1}^{n} y_{i}>\mu(G) \cdot \beta . \\
\sum_{i=1}^{n} \alpha_{i}^{(j)} y_{i} \leqq \beta, \quad 1 \leqq j \leqq s .
\end{gathered}
$$

Using (5) and (6) we shall construct a graph $H$ for which the inequality

$$
\mu(G \times H)>\mu(H) \cdot \mu(G)
$$

will hold. This of course will complete the proof of our theorem.

Let $A_{i}, 1 \leqq i \leqq n$, be a family of $n$ disjoint sets such that card $A_{i}$ $=y_{i}$. Let Ver $H=\bigcup_{i=1}^{n} A_{i}$. Two vertices in $H: z, u$ will be joined by an edge if:
(a) $z=u$
(b) $z \in A_{i}, \quad u \in A_{j} \rightarrow i \neq j$ and $g_{i} \neq g_{j}$.

(Hence any set $A_{i}$ is independent.)

Let $U=\left\{u_{1} \cdots u_{r}\right\}$ be an independent set of vertices in $H$. We may assume that $U \cap A_{i} \neq \varnothing, 1 \leqq i \leqq t$, and $U \cap A_{i}=\varnothing, i>t$. Since $U$ is independent so is $\bigcup_{i=1}^{t} A_{i}$. It follows from our definition of $H$ that the set $\left\{g_{1} \cdots g_{t}\right\}$ is a complete subgraph of $G$ and therefore it is contained in a clique of $G$. Hence we have: $\sum_{i=1}^{t} x_{i} \leqq 1$. But this implies: $\sum_{i=1}^{t} y_{i} \leqq \beta \Rightarrow$ card $\left\{\bigcup_{i=1}^{t} A_{i}\right\} \leqq \beta$. This means that:

$$
\mu(H) \leqq \beta .
$$

Let $D=\left\{\left(g_{i} h\right) \mid h \in A_{i}\right\}(D \subset G \times H)$. If $(g, h),\left(g^{\prime} h^{\prime}\right) \in D$, then $g \rightarrow g^{\prime}$ $\Rightarrow h \rightarrow h^{\prime}$ and therefore $(g h) \rightarrow\left(g^{\prime} h^{\prime}\right)$; if $g \rightarrow g^{\prime}$ it is obvious that $(g h)$ $\rightarrow\left(g^{\prime} h^{\prime}\right)$ i.e. $D$ is an independent set of vertices in $G \times H$. Now using (5), (6) and (7) we obtain: 


$$
\mu(G \times H) \geqq \operatorname{card} D=\operatorname{card}\left\{\bigcup_{i=1}^{n} A_{i}\right\}=\sum_{i=1}^{n} y_{i}>\mu(G) \cdot \beta \geqq \mu(G) \cdot \mu(H) .
$$

This completes the proof of our theorem.

REMARKs. The condition of Theorem 2 can be expressed in terms of discrete linear programming as follows: $G$ is universal if and only if for any set of nonnegative integers $x_{i}$ satisfying:

$$
\sum_{i=1}^{n} \alpha_{i}^{(j)} x_{i} \leqq \beta, \quad 1 \leqq j \leqq s, \quad \sum_{i=1}^{n} x_{i} \leqq \mu(G) \cdot \beta
$$

for all nonnegative integers $\beta$.

Suppose $G$ is not universal, i.e. there exists a $\beta$ for which $\max \sum_{i=1}^{n} x_{i}>\mu(G) \cdot \beta$. If $\left\{g_{1} \cdots g_{\mu(G)}\right\}$ is an independent set of vertices in $G$, choose $y_{i}=x_{i}+1(1 \leqq i \leqq \mu(G)), y_{i}=x_{i}(i>\mu(G))$; it is obvious that $\sum_{i=1}^{n} \alpha_{i}^{(j)} y_{i} \leqq \beta+1$ while $\sum_{i=1}^{n} y_{i}>\mu(G) \cdot(\beta+1)$. This shows that if $G$ is not universal with respect to $\beta$ it is also not universal with respect to $\beta+1$. Since the number of different graphs (up to isomorphism) with $n$ vertices is finite, it follows that there exists an in teger $\beta(n)$ such that $G$ is universal if and only if $G$ is universal with respect to $\beta(n)$. The function $\beta(n)$ is a nondecreasing function of $n$. The values of $\beta(n)$ for $n \leqq 5$ may be easily computed using Shannon's observation [3] that all graphs with at most 5 vertices are universal except for the pentagon which is not universal with respect to 2 . Hence $\beta(n)=0, n \leqq 4, \beta(5)=2$. Using (iii) in the proof of Theorem 2 one can see that $\beta(n)<n$ !.

One can use Theorem 2 to estimate the value of $\mu(G \times H)$ as follows: given $G$ and $H$ one can calculate

subject to

$$
a=\max \sum_{i=1}^{n} x_{i}
$$

$$
\sum_{i=1}^{n} \alpha_{i}^{(j)} x_{i} \leqq \mu(H), \quad 1 \leqq j \leqq s_{G}
$$

where $x_{i}$ is a nonnegative integer and

$$
b=\max \sum_{i=1}^{m} y_{i}, \quad \sum_{i=1}^{m} \beta_{i}^{(j)} y_{i} \leqq \mu(G) ; \quad i \leqq j \leqq s_{H} .
$$

$\left(\beta_{i}^{(j)}\right.$ has the same meaning with respect to $H$ as $\alpha_{i}^{(j)}$ with respect to $G$.) It is obvious that: $\mu(G \times H) \leqq \min (a, b)$.

4. In this paragraph we shall show that Shannon's condition is not necessary. Observe first that if $G$ is a graph and $\sigma$ a preserving function defined on $G$, and if $A C G$ is an independent set of vertices, then 
$\sigma(A)$ is independent and $\operatorname{card}\{\sigma(A)\}=$ card $A$; therefore we always have $\mu(\sigma(G))=\mu(G)$. Since $\sigma^{-1}(g)$ is a complete subgraph of $G$ it follows that $G$ is covered by card $\{\operatorname{Ver} \sigma(G)\}$ complete subgraphs, therefore a necessary condition for the existence of a preserving function $\sigma$ such that $\sigma(G)$ is independent is that $G$ is covered by $\mu(G)$ complete subgraphs.

Let $G_{1}$ and $G_{2}$ be two disjoint pentagons and $G_{3}$ a set of 5 vertices no one of which belongs to $G_{1}$ or $G_{2}$. Adjoin by an edge each vertex of $G_{3}$ to all the vertices of $G_{1}$ and $G_{2}$. Let $H$ be the graph defined by these relations, hence we have:

$$
\operatorname{card}\{\operatorname{Ver} H\}=15, \mu(H)=5 \quad\left(G_{3} \text { is independent }\right) .
$$

Since a pentagon cannot be covered by less than 3 complete subgraphs, it is obvious that $H$ cannot be covered by less than 6 complete subgraphs. Thus we have shown that Shannon's condition cannot hold for $H$.

To show that $H$ is universal observe that all the cliques of $H$ are triangles, every vertex of $H$ is contained in exactly 10 different cliques and the number of different cliques is 50 , therefore the following holds:

$$
1 \leqq j \leqq 50, \quad \sum_{i=1}^{15} \alpha_{i}^{(j)} x_{i} \leqq 1 \Rightarrow \sum_{j=1}^{50} \sum_{i=1}^{15} \alpha_{i}^{(j)} x_{i} \leqq 50,
$$

but:

$$
\sum_{j=1}^{50} \sum_{i=1}^{15} \alpha_{i}^{(j)} x_{i}=10 \sum_{i=1}^{15} x_{i} \leqq 50 \Rightarrow \max \sum_{i=1}^{15} x_{i} \leqq 5=\mu(H)
$$

and, by Theorem $2, H$ is universal.

Q.E.D.

\section{REFERENCES}

1. C. Berge, Théorie des graphes et ses applications, Dunod, Paris, 1958.

2. O. Ore, Theory of graphs, Amer. Math. Soc. Colloq. Publ., Vol. 38, Amer. Math. Soc., Providence, R. I., 1962.

3. C. E. Shannon, The zero error capacity of a noisy channel, I.R.E., Transactions on Information Theory, IT-2, 1956.

Hebrew UNIVERSITy 